\title{
Brain metastases management paradigm shift: A case report and review of the literature
}

\author{
TAMER REFAAT $^{1-3}$, SEAN SACHDEV ${ }^{1}$, BRIJAL DESAI ${ }^{1}$, IAN BACCHUS $^{1}$, SALEH HATOUM $^{1}$, PLATO LEE $^{1}$, \\ ORIN BLOCH ${ }^{4}$, JAMES P. CHANDLER ${ }^{4}$, JOHN KALAPURAKAL ${ }^{1}$ and MARYANNE HOFFMAN MARYMONT ${ }^{1}$ \\ ${ }^{1}$ Department of Radiation Oncology, Robert H. Lurie Comprehensive Cancer Center, Feinberg School of Medicine, \\ Northwestern University, Chicago, IL 606611, USA; ${ }^{2}$ Department of Clinical Oncology and Nuclear Medicine, Faculty of \\ Medicine, Alexandria University, Alexandria 21561, Egypt; ${ }^{3}$ Northwestern Medicine Developmental Therapeutics Institute \\ (NMDTI); ${ }^{4}$ Department of Neurological Surgery, Robert H. Lurie Comprehensive Cancer Center, \\ Feinberg School of Medicine, Northwestern University, Chicago, IL 606611, USA
}

Received May 12, 2015; Accepted October 22, 2015

DOI: $10.3892 / \mathrm{mco} .2016 .772$

\begin{abstract}
Brain metastases are the most common intracranial tumors in adults, accounting for over half of all lesions. Whole-brain radiation therapy (WBRT) has been a cornerstone in the management of brain metastases for decades. Recently, stereotactic radiosurgery (SRS) has been considered as a definitive or postoperative approach instead of WBRT, to minimize the risk of cognitive impairment that may be associated with WBRT. This is the case report of a 74-year-old female patient who was diagnosed with lung cancer in November, 2002, and histopathologically confirmed brain metastases in January, 2005. The patient received 5 treatments with Gamma Knife SRS for recurring brain metastases between 2005 and 2014. The patient remains highly functional, with stable intracranial disease at 10 years since first developing brain metastases, and with stable lung disease. Therefore, Gamma Knife SRS is a safe and effective treatment modality for patients with recurrent intracranial metastases, with durable local control and minimal cognitive impairment.
\end{abstract}

\section{Introduction}

Brain metastases are the most common intracranial tumors in adults, accounting for over half of all lesions $(1,2)$. Whole-brain radiation therapy (WBRT) has been a cornerstone in the management for brain metastases for decades (3-6). Modern surgical techniques that minimize surgical morbidity and mortality have made resection a viable option for a larger number of patients, including providing superior outcomes for patients with 1-3 metastases and those with symptomatic

Correspondence to: Dr Maryanne Hoffman Marymont, Department of Radiation Oncology, Robert H. Lurie Comprehensive Cancer Center, Feinberg School of Medicine, Northwestern University, 676 North St. Clair, Suite 1820, Chicago, IL 606611, USA E-mail: mmarymon@nm.org

Key words: brain metastases, radiosurgery, Gamma Knife disease (7). Recently, stereotactic radiosurgery (SRS) has been considered as a definitive or postoperative approach instead of WBRT, to minimize the risk of cognitive impairment that may be associated with WBRT (8).

\section{Case report}

A 74-year-old Caucasian woman was diagnosed with a right upper lung lesion, for which she underwent a right upper lobectomy on November 7, 2002. The histopathological evaluation revealed an adenocarcinoma [American Joint Committee on Cancer stage I (T2N0M0), grade 3, measuring 3.5x3.0x3.0 cm] involving the bronchial margin. The adjacent lung parenchyma exhibited emphysematous changes but no tumor involvement; all the resected lymph nodes were free of disease. After 4 cycles of adjuvant chemotherapy with paclitaxel and carboplatin, the patient was closely followed up, including interval physical evaluations and regular imaging studies, which included computed tomography (CT) and positron emission tomography (PET) scans. The patient was free of disease at her last imaging studies on December 20, 2004.

On January 27, 2005, the patient tripped and fell while walking up a staircase; she denied suffering from headaches, confusion, dizziness or visual disturbances and was subsequently treated for a right arm fracture. On March 15, 2005, the patient presented with altered mental status; an MRI scan of the brain performed on the same day revealed a large enhancing right frontal lobe mass, measuring 3.3x3.0x3.1 cm in the anteroposterior, transverse and craniocaudal dimensions, respectively. The lesion was associated with extensive surrounding edema in the right frontal lobe, causing a mass effect on the genu of the corpus callosum and adjacent left frontal lobe. There was a second enhancing focus, measuring $6 \mathrm{~mm}$, within the vermis. No other abnormal parenchymal enhancement was identified. Due to the significant edema associated with the frontal lesion and the altered mental status, the patient underwent a right frontal craniotomy and tumor resection and fared well postoperatively. The histopathological evaluation revealed a fairly well-differentiated metastatic adenocarcinoma; the immunohistochemical staining results 
were positive for cytokeratin $(\mathrm{CK}) 7$ and thyroid transcription factor-1 and negative for CK 20 and thyroglobulin, which were compatible with metastasis from a primary lung tumor.

After consenting to the procedure, the patient was scheduled for postoperative Gamma Knife SRS. On April 1, 2005, following placement of a stereotactic frame with local anesthetic, the patient was positioned supine on an MR gantry for a double-contrast scan. Relevant images were selected and transferred to the Gamma Knife planning station for treatment planning. Following a neuroradiology review, no additional lesions were identified; only the postoperative residual cavity in the right frontal region and the vermian metastasis were noted on the pre-procedure MRI. The matrices were set over the target areas. The initial frontal lesion was planned out using 15 isocenters with a combination of 4-, 8- and 18-mm collimators with a total volume of $\sim 10.2 \mathrm{~cm}^{3}$. A dose of $16 \mathrm{~Gy}$ was selected, prescribed to the $50 \%$ isodose line. A single isocenter with $8-\mathrm{mm}$ collimator was used for the vermian lesion (with a volume of $0.6 \mathrm{~cm}^{3}$ ) and a dose of $20 \mathrm{~Gy}$ was selected to the $50 \%$ isodose line. The patient tolerated the procedure well.

The patient was again closely followed up with serial clinical evaluations and imaging; she fared well post-SRS and did not experience headaches or any other neurological complaints. The follow-up brain MRIs, performed every 3 months, revealed good control of the frontal lobe cavity and the vermian lesion, with no additional new lesions.

After 8 months (December 9, 2005), on a follow-up brain MRI, a 4-mm enhancing focus was identified in the left posterior parietal subcortical white matter, with minimal associated FLAIR signal abnormality. This lesion was new and considered to be consistent with a metastatic focus. Otherwise, there continued to be no evidence of local recurrence or metastatic disease progression systemically, apart from the new brain lesion. The patient underwent a second Gamma Knife SRS treatment on December 29, 2005. The pre-SRS MRI reviewed by the neuroradiologist only showed the metastatic lesion that was treated. The previously treated cerebellar lesion had largely disappeared; the frontal lesion remained small and stable, with an excellent collapse of the cavity. Based on this information, a treatment plan was generated using an 8-mm collimator over the left parietal occipital metastatic lesion, with 4 isocenters circumferentially covering the metastatic lesion. Based on the previous excellent response to $20 \mathrm{~Gy}$ for a single metastatic lesion, 20 Gy was again selected (Fig. 1).

The patient was again closely followed up. All the physical examinations and imaging studies revealed no disease progression until April 23, 2007 (16 months after the second SRS treatment). At that time, although the patient remained clinically asymptomatic, a brain MRI revealed the development of two new metastatic lesions: One within the left precentral gyrus, and another in the posterior occipital area. A follow-up MRI on July 8, 2007 revealed interval development of enhancement in the high left posterior frontal lobe, along with persistence of the lesions seen in the previous scan. Based on the patient's good control and response to the previous Gamma Knife treatments and the fact that the brain disease remained focal, the patient, the neurosurgeon and the radiation oncologist concurred in selecting Gamma Knife SRS over whole-brain radiation therapy (WBRT). On August 7, 2007 (20 months after the second SRS treatment and 28 months since being first diagnosed with brain metastases), the patient received a third Gamma Knife SRS treatment.

Following this treatment, the patient's serial MRI scans revealed completely stable intracranial disease (with additional scans revealing no lung disease or extracranial progression) up to September 13, 2010, when a follow-up brain MRI revealed progression of five intracranial lesions. As the patient continued to fare well clinically, a repeat brain MRI on April 11,2011 revealed a marginal increase in the size of the left parietal metastatic lesion, but a stable appearance of the other metastatic lesions. The patient was offered WBRT vs. repeat Gamma Knife SRS; after a thorough discussion of the risks and benefits, she consented to a repeat course of SRS and received her fourth Gamma Knife SRS on May 13, 2011 (45 months after the third SRS treatment and 71 months since being diagnosed with brain metastases) to the one lesion that was known to be new and progressing in the right parietal area; this was performed utilizing 2 isocenters with a total of 20 Gy prescribed to the $50 \%$ isodose line (Fig. 2).

Notably, the patient's lung disease remained controlled up to October, 2011; at that time, although she remained asymptomatic, a follow-up CT demonstrated occlusion of the bronchus of the remaining portion of the right lung and soft tissue density suggestive of a recurrence. A subsequent PET/CT scan confirmed prominent uptake within this region, with no other evidence of metastatic disease. The patient received a course of palliative radiation therapy of $30 \mathrm{~Gy}$ in 10 fractions to the progressive chest disease, which was completed on December 14, 2011, followed by initiation of targeted therapy with erlotinib.

The patient remained highly functional, without significant neurological symptoms and with largely stable intracranial disease until October, 2014 (>10 years since the first diagnosis of brain metastasis), at which point she had five growing brain lesions that were associated with vasogenic edema and local mass effect. On October 23, 2014 (41 months after the fourth SRS treatment and 112 months since being diagnosed with brain metastases), the patient, then aged 84 years, received her fifth Gamma Knife SRS: The first lesion in the right frontal lobe (with a total volume of $0.57 \mathrm{~cm}^{3}$ ) was targeted with $18 \mathrm{~Gy}$ prescribed to the $50 \%$ isodose line, covering $100 \%$ of the target; the second lesion in the left atrial region (with a total volume of $0.29 \mathrm{~cm}^{3}$ ) was targeted with $18 \mathrm{~Gy}$ prescribed to the $50 \%$ isodose line, covering $100 \%$ of the target; the third lesion in the right frontal lobe (with a total volume of $0.57 \mathrm{~cm}^{3}$ ) was targeted with 18 Gy prescribed to the $50 \%$ isodose line, covering $100 \%$ of the target; the fourth lesion in the left temporal lobe (with a total volume of $1.4 \mathrm{~cm}^{3}$ ) was targeted with 16 Gy prescribed to the $50 \%$ isodose line, covering $100 \%$ of the target; and the 5th lesion in the cerebellar vermis (with a total volume of $0.09 \mathrm{~cm}^{3}$ ) was targeted with a dose of $20 \mathrm{~Gy}$ prescribed to the $50 \%$ isodose line, covering $100 \%$ of the target (Fig. 3). The patient again tolerated the treatment well and without complications.

On the last follow-up on November 17,2014, before this case report was submitted, the patient remained highly functional, with stable intracranial disease 10.5 years since first developing brain metastases, and with stable lung disease. The patient occasionally noted problems with her energy levels; however, she had no other health complaints, no sleep disturbances and 


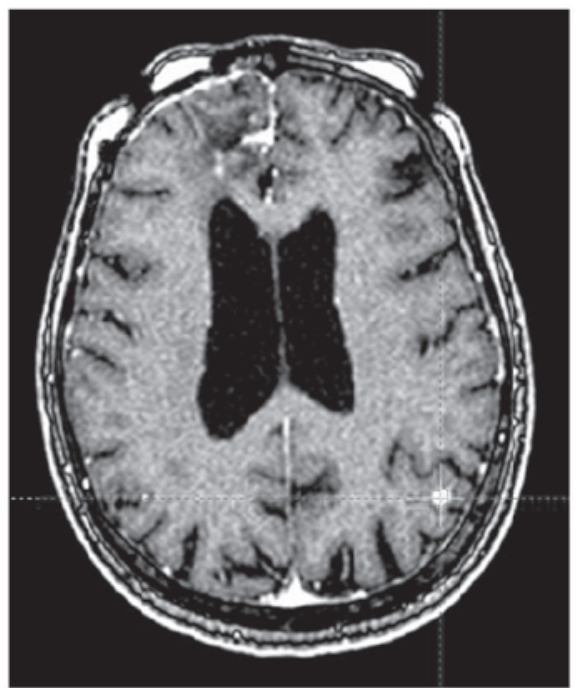

Figure 1. Second Gamma Knife treatment: 20 Gy for a single left posterior parietal metastatic lesion.

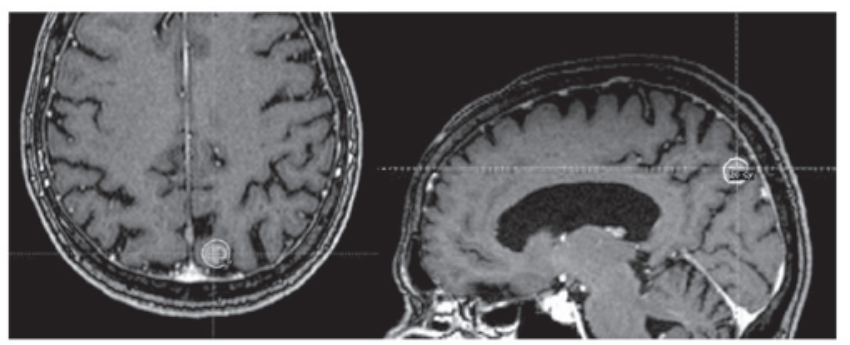

Figure 2. Fourth Gamma Knife Treatment: 20 Gy for a single right parietal metastatic lesion.

no neurological symptoms, with the exception of occasional headaches. The patient reported no dizziness, confusion, declining mentation or any other neurological issues.

\section{Discussion}

The cumulative incidence of brain metastases in lung cancer patients has been reported in two series: One from the Metropolitan Detroit Cancer Surveillance System, reporting the highest incidence in primary lung cancer patients $(19.9 \%)$ compared with melanoma $(6.9 \%)$, renal $(6.5 \%)$, breast $(5.1 \%)$ and colorectal $(1.8 \%)$ primary cancers $(9)$; and the other from a Dutch series, reporting a 5-year cumulative incidence of 7.4, $16.3,9.8,5.0$ and $1.2 \%$ in patients with melanoma and lung, renal, breast and colorectal carcinoma, respectively (10).

The Recursive Partitioning Analysis (RPA) developed by the Radiation Therapy Oncology Group (RTOG) likely remains the most reliable and widely used prognostic index for brain metastases. The RPA divides patients with brain metastases into 3 classes as follows: Class I (best survival) are patients with a Karnofsky performance status (KPS) of $\geq 70$, aged $<65$ years, with a controlled primary tumor, and no other systemic metastases; class III (worst survival) includes patients with a KPS $<70$; these patients have a median survival of only 2.3 months; finally class II includes all patients intermediate between classes I and III, with a median survival of
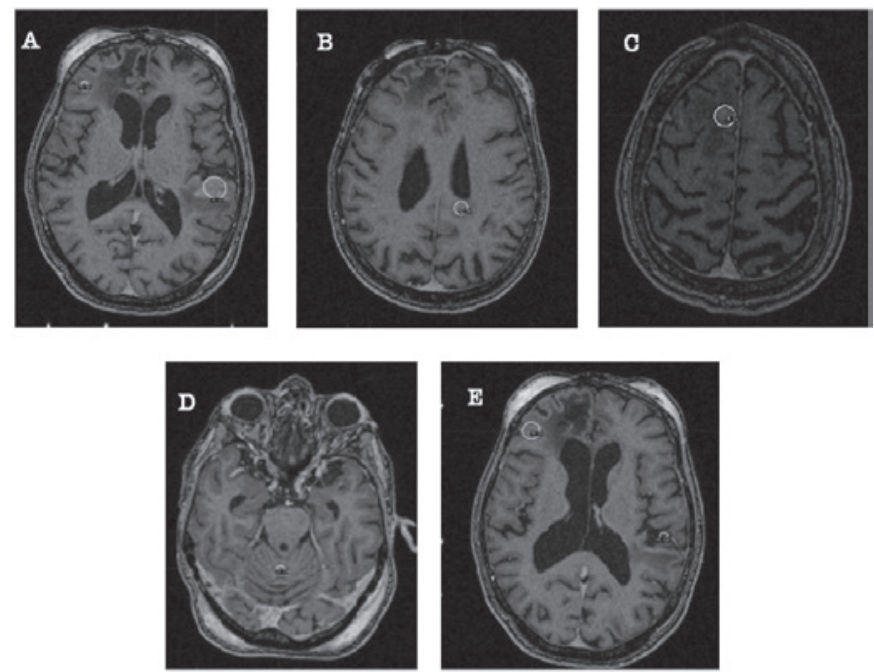

Figure 3. Fifth Gamma Knife Treatment: (A) The first lesion in the left temporal lobe with a total volume of $1.4 \mathrm{~cm}^{3}$ was targeted with 16 Gy prescribed to the $50 \%$ isodose line, covering $100 \%$ of the target. (B) The second lesion in the left atrial region with a total volume of $0.29 \mathrm{~cm}^{3}$ was targeted with 18 Gy prescribed to the $50 \%$ isodose line, covering $100 \%$ of the target. (C) The third lesion in the right frontal lobe with a total volume of $0.57 \mathrm{~cm}^{3}$ was targeted with 18 Gy prescribed to the $50 \%$ isodose line, covering $100 \%$ of the target. (D) The fourth lesion in the cerebellar vermis with a total volume of $0.09 \mathrm{~cm}^{3}$ was targeted with a prescription dose of $20 \mathrm{~Gy}$ prescribed to the $50 \%$ isodose line, covering $100 \%$ of the target. (E) The fifth lesion in the right frontal lobe with a total volume of $0.57 \mathrm{~cm}^{3}$ was targeted with $18 \mathrm{~Gy}$ prescribed to the $50 \%$ isodose line, covering $100 \%$ of the target.

4.2 months (11). Based on the RPA, our patient belonged to class II at presentation, with an expected median survival of 4.2 months had she received WBRT. The RPA did not account for the primary diagnosis; instead, the diagnosis-specific graded prognostic assessment (DS-GPA) was developed later, based on a retrospective analysis of 4,259 patients with consideration of the primary diagnosis (12). Based on DS-GPA, our patient's score at presentation was 2.5 , with an estimated median survival of 9.4 months.

A number of RTOG studies have attempted to identify the optimal dose and fractionation for WBRT in the setting of brain metastases, including RTOG 6901, 7361 and 7601. The cumulative experience obtained from these RTOG studies revealed that: i) 30 Gy in 10 fractions is the most widely accepted, tolerable and effective fractionation in this patient subset; ii) $20 \mathrm{~Gy}$ in 5 fractions, 30 Gy in 10 fractions, 30 Gy in 15 fractions, $40 \mathrm{~Gy}$ in 15 fractions, 40 Gy in 20 fractions and 50 Gy in 20 fractions did not achieve superior results with respect to palliation of the symptoms, median time to progression, cause of death or median survival; and iii) ultra-short fractionation (10 Gy in 1 fraction and 12 Gy in 2 fractions) is not as effective as standard fractionation (20-30 or 40 Gy in 1-4 weeks) for palliation of patients with brain metastases (3-6).

Surgery is now widely applied, particularly in patients with 1-3 brain metastases, aiming primarily to provide prompt symptomatic relief, obtain histological diagnosis and possibly improve local control. With the application of modern surgical techniques, the morbidity and mortality have been significantly reduced and the option of resection has become feasible for a larger number of patients, including those with lesions in eloquent as well as non-eloquent regions of the 
brain (7). An overall survival (OS) advantage has also been reported in two randomized clinical trials comparing surgery plus WBRT vs. WBRT alone. Patchell et al (13) randomized 48 single-brain metastasis patients to either WBRT or surgery followed by WBRT (25 in the surgical group and 23 in the radiation group). Patients who had undergone surgical resection exhibited a significant OS improvement compared with the WBRT alone patients (40 vs. 15 weeks, respectively; $\mathrm{P}<0.01)$. Furthermore, surgical patients had fewer recurrences in the brain, and a better quality of life compared with WBRT alone patients. Noordijk et al (14) randomized 66 patients with a single brain metastasis to surgery plus WBRT vs. WBRT alone. The patients were stratified for lung cancer vs. other primary sites and for progressive vs. stable systemic disease. The study reported that, among 63 evaluable patients, surgery plus WBRT achieved a longer OS (median, 10 vs. 6 months, respectively; $\mathrm{P}=0.04$ ). The largest difference between the two treatment arms was observed in patients with inactive extracranial disease (median, 12 vs. 7 months, respectively; $\mathrm{P}=0.02$ ). Patients with active extracranial disease had an equal median OS of 5 months, regardless of the treatment modality. Patients who were randomized to surgery and WBRT remained functionally independent for a longer period of time. In addition, patients aged $>60$ years had a hazard ratio of $2.74(\mathrm{P}=0.003)$ for death compared with younger patients. The majority of the patients succumbed to to systemic disease progression (14).

Stereotactic radiosurgery (SRS) utilizes multiple convergent beams to deliver high-precision radiation to a defined target volume with a rapid dose fall-off, with the ultimate goal of delivering a high dose per fraction to the target volume and minimal radiation to the surrounding clinical structures; this requires high precision for localization of the target volume, as well as patient positioning during treatment. In our institution, SRS is delivered by the Leksell Gamma Knife Perfexion system (Elekta AB, Stockholm, Sweden) that uses 192 cobalt-60 gamma ray sources spatially oriented so that all the beams converge at a single point (isocenter). The Gamma Knife is able to provide a target accuracy of 0.1-1 mm (15). Other methods of delivering SRS include high-energy X-rays produced by linear accelerators, or even charged particles, such as protons produced by cyclotrons $(16,17)$.

Initially, SRS was investigated as a boost after WBRT in patients with 1-3 newly diagnosed brain metastases. RTOG 9508 demonstrated superior survival (6.5 vs. 4.9 months), a higher response rate after 3 months of treatment and better local control after 1 year of treatment (82 vs. 71\%) for patients receiving a radiosurgery boost (18). However, an increasing number of studies (none within the context of randomized clinical trials) reported that SRS may be as effective as surgery, with comparable local control (19-22). Yamamoto et al (23) recently reported the results of a study which enrolled patients with 1-10 newly diagnosed brain metastases (the largest tumor was $<10 \mathrm{ml}$ in volume and $<3 \mathrm{~cm}$ in longest diameter; the total cumulative volume was $\leq 15 \mathrm{ml}$ ) and a KPS score of $\geq 70$ from 23 facilities in Japan; the results of the study suggested that SRS without WBRT in patients with 5-10 brain metastases is non-inferior to treatment in patients with 2-4 brain metastases and may be a suitable alternative for WBRT in patients with $\leq 10$ brain metastases (23). The EORTC 22952-26001 study included
359 patients with 1-3 brain metastases who underwent SRS or surgery, with 100 patients allocated to obsrvation and 99 allocated to adjuvant WBRT. While adjuvant WBRT reduced intracranial relapses and neurological deaths, it failed to improve the OS or the duration of functional independence (24).

The treatment approach to brain metastases has been evolving over the last couple of decades, with advances in diagnostic radiology, radiation oncology technology, surgical techniques and systemic therapies, all leading to longer survival. Therefore, several institutions are selecting SRS as a definitive or postoperative approach over WBRT to minimize the risk of cognitive decline (8). Asher et al recently proposed a new treatment paradigm, namely neoadjuvant SRS followed by surgical resection. A total of 47 patients with a total of 51 brain metastatic lesions received neoadjuvant SRS followed by surgical resection. The 1-year OS and local control rates were 60 and $85.6 \%$, respectively. That study concluded that neoadjuvant SRS is a safe and effective modality meriting consideration in a multi-institutional trial (8).

SRS will likely continue to play a significant role in the treatment of brain metastases in the future, possibly with even more improved patient outcomes. Indeed, as our case demonstrates, with appropriate use of this powerful modality in carefully selected patients (possibly treated with a tailored multidisciplinary care plan of targeted agents, surgery and systemic therapy), there may be further improvement in the outcomes of patients presenting with a clinical challenge such as metastatic intracranial disease.

In conclusion, Gamma Knife SRS is a safe and effective treatment modality for patients with recurrent intracranial metastasis, with durable local control and minimal cognitive impairment.

\section{Acknowledgements}

To the memory of Christopher C. Getch, MD (1961-2012), the treating neurosurgeon for this case who performed the patient's surgery, follow-up assessments and first four Gamma Knife SRS treatments, and passed away unexpectedly on January 9, 2012. To the memory of Andrew Parsa, MD (1967-2015), Chairman of Neurological Surgery at Northwestern University's Feinberg School of Medicine, who passed away unexpectedly on April 13, 2015.

\section{References}

1. Wen PY and Loeffler JS: Management of brain metastases. Oncology (Williston Park) 13: 941-954, 957-61; discussion 961-962, 1999.

2. Johnson JD and Young B: Demographics of brain metastasis. Neurosurg Clin N Am 7: 337-344, 1996.

3. Borgelt B, Gelber R, Kramer S, Brady LW, Chang CH, Davis LW, Perez CA and Hendrickson FR: The palliation of brain metastases: Final results of the first two studies by the Radiation Therapy Oncology Group. Int J Radiat Oncol Biol Phys 6: 1-9, 1980.

4. Kurtz JM, Gelber R, Brady LW, Carella RJ and Cooper JS: The palliation of brain metastases in a favorable patient population: A randomized clinical trial by the Radiation Therapy Oncology Group. Int J Radiat Oncol Biol Phys 7: 891-895, 1981.

5. Gelber RD, Larson M, Borgelt BB and Kramer S: Equivalence of radiation schedules for the palliative treatment of brain metastases in patients with favorable prognosis. Cancer 48: 1749-1753, 1981. 
6. Borgelt B, Gelber R, Larson M, Hendrickson F, Griffin T and Roth R: Ultra-rapid high dose irradiation schedules for the palliation of brain metastases: Final results of the first two studies by the Radiation Therapy Oncology Group. Int J Radiat Oncol Biol Phys 7: 1633-1638, 1981.

7. Sawaya R, Hammoud M, Schoppa D, Hess KR, Wu SZ, Shi WM and Wildrick DM: Neurosurgical outcomes in a modern series of 400 craniotomies for treatment of parenchymal tumors. Neurosurgery 42: 1044-55; discussion 1055-1056, 1998.

8. Asher AL, Burri SH, Wiggins WF, Kelly RP, Boltes MO, Mehrlich M, Norton HJ and Fraser RW: A new treatment paradigm: Neoadjuvant radiosurgery before surgical resection of brain metastases with analysis of local tumor recurrence. Int J Radiat Oncol Biol Phys 88: 899-906, 2014.

9. Barnholtz-Sloan JS, Sloan AE, Davis FG, Vigneau FD, Lai P and Sawaya RE: Incidence proportions of brain metastases in patients diagnosed (1973 to 2001) in the Metropolitan Detroit Cancer Surveillance System. J Clin Oncol 22: 2865-2872, 2004.

10. Schouten LJ, Rutten J, Huveneers HA and Twijnstra A: Incidence of brain metastases in a cohort of patients with carcinoma of the breast, colon, kidney and lung and melanoma. Cancer 94: 2698-2705, 2002.

11. Gaspar L, Scott C, Rotman M, Asbell S, Phillips T, Wasserman T, McKenna WG and Byhardt R: Recursive partitioning analysis (RPA) of prognostic factors in three Radiation Therapy Oncology Group (RTOG) brain metastases trials. Int J Radiat Oncol Biol Phys 37: 745-751, 1997.

12. Sperduto PW, Chao ST, Sneed PK, Luo X, Suh J, Roberge D, Bhatt A, Jensen AW, Brown PD, Shih H, et al: Diagnosis-specific prognostic factors, indexes and treatment outcomes for patients with newly diagnosed brain metastases: A multi-institutional analysis of 4,259 patients. Int J Radiat Oncol Biol Phys 77: 655-661, 2010.

13. Patchell RA, Tibbs PA, Walsh JW, Dempsey RJ, Maruyama Y, Kryscio RJ, Markesbery WR, Macdonald JS and Young B: A randomized trial of surgery in the treatment of single metastases to the brain. N Engl J Med 322: 494-500, 1990.

14. Noordijk EM, Vecht CJ, Haaxma-Reiche H, Padberg GW, Voormolen JH, Hoekstra FH, Tans JT, Lambooij N, Metsaars JA and Wattendorff AR: The choice of treatment of single brain metastasis should be based on extracranial tumor activity and age. Int J Radiat Oncol Biol Phys 29: 711-717, 1994.
15. Schwartz M: Stereotactic radiosurgery: Comparing different technologies. CMAJ 158: 625-178, 1998.

16. Kuo JS, Yu C, Petrovich Z and Apuzzo ML: The CyberKnife stereotactic radiosurgery system: Description, installation and an initial evaluation of use and functionality. Neurosurgery 53: 1235-1239; discussion 1239, 2003.

17. Miller DW: A review of proton beam radiation therapy. Med Phys 22: 1943-1954, 1995.

18. Andrews DW, Scott CB, Sperduto PW, Flanders AE, Gaspar LE, Schell MC, Werner-Wasik M, Demas W, Ryu J, Bahary JP, et al: Whole-brain radiation therapy with or without stereotactic radiosurgery boost for patients with one to three brain metastases: Phase III results of the RTOG 9508 randomised trial. Lancet 363: 1665-1672, 2004.

19. Pirzkall A,Debus J,LohrF,Fuss M, Rhein B,Engenhart-Cabillic R and Wannenmacher M: Radiosurgery alone or in combination with whole-brain radiotherapy for brain metastases. J Clin Oncol 16: 3563-3569, 1998.

20. Alexander E III, Moriarty TM and Loeffler JS: Radiosurgery for metastases. J Neurooncol 27: 279-285, 1996.

21. Alexander E III, Moriarty TM, Davis RB, Wen PY, Fine HA, Black PM, Kooy HM and Loeffler JS: Stereotactic radiosurgery for the definitive, noninvasive treatment of brain metastases. J Natl Cancer Inst 87: 34-40, 1995.

22. Flickinger JC, Kondziolka D, Lunsford LD, Coffey RJ, Goodman ML, Shaw EG, Hudgins WR, Weiner R, Harsh GR IV and Sneed PK: A multi-institutional experience with stereotactic radiosurgery for solitary brain metastasis. Int J Radiat Oncol Biol Phys 28: 797-802, 1994 .

23. Yamamoto M, Serizawa T, Shuto T, Akabane A, Higuchi Y, Kawagishi J, Yamanaka K, Sato Y, Jokura H, Yomo S, et al: Stereotactic radiosurgery for patients with multiple brain metastases (JLGK0901): A multi-institutional prospective observational study. Lancet Oncol 15: 387-395, 2014.

24. Kocher M, Soffietti R, Abacioglu U, Villà S, Fauchon F, Baumert BG, Fariselli L, Tzuk-Shina T, Kortmann RD, Carrie C, et al: Adjuvant whole-brain radiotherapy versus observation after radiosurgery or surgical resection of one to three cerebral metastases: Results of the EORTC 22952-26001 study. J Clin Oncol 29: 134-141, 2011. 